\title{
Prognosis and Complications of Hyperuricemia Patients Undergoing Isolated Coronary Artery Bypass Surgery
}

\author{
Haydar Yasa ${ }^{1,2^{*}}$ \\ ${ }^{1}$ Evliya Celebi Education and Research Hospital, Health Science University, Turkey \\ ${ }^{2}$ Bati Anadolu Central Hospital, Turkey
}

\begin{abstract}
Abbreviations
AMI: Acute myocardial infarction; BMI: Body mass index; CABG: Coronary artery bypass grafting; CHF: Congestive heart failure; CKD: Chronic kidney disease; CVD: Cardiovascular disease; DM: Diabetes mellitus; HTN: Hypertension; IOT-Re: Reintubation rates
\end{abstract}

\section{Introduction}

Recent studies suggest that high levels of serum uric acid of very early life are a result of the in-utero environment and may lead to elevated blood pressure in adulthood. However, serum uric acid levels can change throughout life. Researchers investigated the effect of serum uric acid levels in childhood on the blood pressure tracking and analysed blood pressure according to changes in serum uric acid levels in early life.

Hyperuricemia is associated with deleterious effects on endothelial dysfunction, oxidative metabolism, platelet adhesiveness, hemorheology, and aggregation. The atherosclerotic plaque contains a considerable amount of uric acid which may increase platelet adhesiveness and potentiate thrombus formation [1].

There is also increasing evidence that elevated serum uric acid levels may predict the development of type 2 diabetes [2].

Despite the association of hyperuricemia with cardiovascular risk factors, it has remained controversial as to whether uric acid is an independent predictor of cardiovascular disease, with many studies in favour [3-7] and others against $[8,9]$.

Serum concentrations of uric acid, the end product of the metabolism of purine compounds, above $7 \mathrm{mg} / \mathrm{dL}$ result in hyperuricemia, causally associated with gout as evidenced in randomized clinical trials using urate lowering therapies [9].

One of the major causes of hypertension and negative effects at the same time during childhood is a disease in which the multiplication of those provided in the etiology of Diabetes mellitus is present. It is possible to speculate that the effects of the early onset of the early onset have serious side effects, in particular cardiovascular system, hypertension, kidney function disorder, diabetes mellitus, and calcium turnover problems. There is insufficient study of the effects of Hyperuricemia on coronary bypass surgery. The aim of our study was therefore to assess the operative and short-term prognosis of hyperurisemic who had CABG (coronary artery bypass graft), comparing non-hyeprurisemic patients. The results of this study are expected to enhance our knowledge regarding the role of uric acid level on outcomes of patients with coronary bypass surgery and thus can be use by clinician in determining patient's prognosis.

\section{Patients and Methods}

Patient evaluation was performed by collecting prospectivedata on patients undergoing CABG at our hospital from March 2012 to May 2017, primary CABG was performed on 86 consecutive patients in the same surgical groups. One senior nurse at the hospital was trained to collect these data using prespecified settings. From this sample, patients who underwent isolated CABG were selected, and this population was then divided into those with hyperurisemia (gruop I) and those without hyperurisemia (group II).

The excluded criteria; Patients were excluded if they had undergone any othercardiac surgery, Diabetes Mellitus Tip I, II, redo CABG, emergency cases, chronic obstructive lung disease, Left ventricular ejection Fraction $\leq 30 \%$, chronic renal disease, active gout attack and symptomatic patients (blood

*Corresponding author: Haydar Yasa, Bat Anadolu Central Hospital, Bayraklı-Izmir, Turkey, Tel: 0902323416767-1084

Accepted: July 13, 2020

Published online: July 15, 2020

Citation: Yasa H (2020) Prognosis and Complications of Hyperuricemia Patients Undergoing Isolated Coronary Artery Bypass Surgery. J Cardiothorac Surg Ther 2020:4(1):61-60 
Citation: Yasa H (2020) Prognosis and Complications of Hyperuricemia Patients Undergoing Isolated Coronary Artery Bypass Surgery. J Cardiothorac Surg Ther 2020:4(1):61-60

uric acid level $\geq 13 \mathrm{mg} / \mathrm{dl}$ ). Patients have demographics, indications for surgery, severity of disease and clinical outcomes for the two groups were shown in Table 1. Baseline clinical data, including medical history, cardiac risk factors, operative details, New York Heart Association functional class, and the European System for Cardiac Operative Risk Evaluation (EurOSCORE), 42 were collected prospectively by an experienced full-time data collector. Serum samples were collected a median of 1 day (interquartile range 1-2 days) preoperatively, and uric acid measured using the ADVIA 1650 General Chemistry Analyzer (Siemens Diagnostics Solutions, Tarrytown, $\mathrm{NY}$ ). Hyperuricemia was defined as $6.8 \mathrm{mg} / \mathrm{dL}$ in men and 6.0 $\mathrm{mg} / \mathrm{dL}$ in women $[9,10]$.

\section{Follow-up}

Postoperative follow-up of patients in the first week, first month were recorded by outpatient visits, phone calls and home visits. Among the clinical complications that occurred following $C A B G$, the following variables were analyzed: Mortalite, $x$-clamp time, total bypas time, perioperative ICU (intensive care unit) times, entubation time, reentubation ratio, postoperative bleeding (drainage), hospitalization time, rehospitalization ratio, neurological complications, pulmonary complications, infectious complications, cardiac arrhythmias, bleeding, wound infection and multiple organ failure occurring within 30 days after the surgery. Postoperative uric acid level measured first visit and second visit. Ethical approval for the study was granted by the local research ethics committee. All of these patients gave their appropriate informed consent and were studied under the approval of the institutional review boards.

\section{Clinical and Uric Acid Data Collection}

Data obtained from the medical records including history taking associated with ischemia symptoms, head to toe physical examination, and laboratory examination. The laboratory examinations include peripheral blood examination, uric acid, blood urea nitrogen (BUN), creatinine, ALT, AST, random blood glucose, electrolyte, lipid profile, cardiac enzyme includes CK-CKMB and troponin T. Other examinations assessed are electrocardiography, chest X-ray, echocardiography, and coronary angiography.

All the hospital's cardiac surgery teams agreed to provide datafor the development of the database. The logistic EuroSCORE was individually calculated for all patients (preoperative risk score) and expressed as the average of the groups.

Table 1: Preoperative demographics.

\begin{tabular}{|c|c|c|c|}
\hline Patients & $\begin{array}{l}\text { Grup I } \\
(n=42)\end{array}$ & $\begin{array}{l}\text { Grup II } \\
(n=44)\end{array}$ & $p$ value \\
\hline Male n (\%) & $33(76.7 \%)$ & $36(71.5 \%)$ & $0.080(2)$ \\
\hline Female $\mathrm{n}(\%)$ & $9(22 \%)$ & $10(25.3 \%)$ & $0.081(2)$ \\
\hline Age mean \pm SD & $67.3 \pm 13.46$ & $8 \pm 14.84$ & $0.087(1)$ \\
\hline PMI n (\%) & $24(56.0)$ & $23(52.9)$ & $0.039(2)$ \\
\hline HLP n (\%) & $65(84.5)$ & 74 (81.4) & $0.799(2)$ \\
\hline HTN n (\%) & $20(46)$ & $16(34.7)$ & $0.044(2)$ \\
\hline PAD (non-surgical) n (\%) & $8(\%)$ & $6(\%)$ & $0.043(2)$ \\
\hline Atrial fibrillation $\mathrm{n}(\%)$ & $2(5.2 \%)$ & none & $0.035(2)$ \\
\hline Smoking n (\%) & $18(40.3 \%)$ & $(38.5 \%)$ & $0.084(2)$ \\
\hline Current smoker. $\mathrm{n}(\%)$ & $15(35.2)$ & $14(32.3)$ & $<0.001(2)$ \\
\hline Alcohol use $\mathrm{n}(\%)$ & $(7.6 \%)$ & $(8.8 \%)$ & $0.671(2)$ \\
\hline Serum creatinine mean \pm SD & $1.2 \pm 0.6$ & $1.0 \pm 0.9$ & $<0.001(1)$ \\
\hline $\mathrm{BMI}\left(\mathrm{kg} / \mathrm{m}^{\prime}\right)$ mean $\pm \mathrm{SD}$ & $24.2 \pm 0.923$ & $1 \pm 1.2$ & $0.565(2)$ \\
\hline Fibrinogen $(\mathrm{mg} / \mathrm{dl})$ mean $\pm \mathrm{SD}$ & $293 \pm 15.4$ & $260 \pm 12.3$ & $0.044(1)$ \\
\hline C-rp (mg/dl) mean \pm SD & $1.2 \pm 0.6$ & $0.8 \pm 0.6$ & $0.034(1)$ \\
\hline \multicolumn{4}{|l|}{ Preoperative ICA ultrasound } \\
\hline ICA velocity $(\mathrm{cm} / \mathrm{sec})$ mean \pm SD & $160 \pm 60$ & $140 \pm 50$ & 0.049 \\
\hline Chronic lung disease. $\mathrm{n}(\%)$ & 9 (21.9) & $8(18.2)$ & $<0.001(2)$ \\
\hline EuroSCORE logistics mean \pm SD & $2.3 \pm 2.0$ & $2.1 \pm 2.4$ & $0.0087(3)$ \\
\hline
\end{tabular}

Pearson correlation's coefficients each pair of variables shown. Asterix denote significiant correlation coefficients $(1 . P \leq 0.05,2$. $P \leq 0.01)$. HTN: Hypertension; CAD: Surgical coronary artery disease; LMCAD: Left main coronary artery disease; LVEF: Left venrikuler ejection fraction; DM: Diabetes mellitus; HLP: Hyperlipoproteinemia; PMI: Previous myocardial infarction. PAD: Peripheral artery disease; BMI: Body mass index; ICA: Internal carotid artery velocity; C-rp: C-rekatif protein); SD: Standart deviation.

(1) Descriptive level of probability determined by the Student's t-test; (2) Descriptive level of probability determined by the chi-square test;

(3) Descriptive level of probability determined by the nonparametric Mann-Whitney $U$ test. 
Citation: Yasa H (2020) Prognosis and Complications of Hyperuricemia Patients Undergoing Isolated Coronary Artery Bypass Surgery. J Cardiothorac Surg Ther 2020:4(1):61-60

\section{Statistical Analysis}

Initially, all variables were descriptively analyzed. For quantitative variables, analyses were carried out by observing minimum and maximum values and calculating the mean and standard deviation. For qualitative variables, absolute and relative frequencies were calculated. To compare the averages of the two groups, we used Student's t-test and when the assumption of data normality was rejected, we used the nonparametric Mann-Whitney $U$ test. To test the homogeneity between proportions, we used the chi-square test or the Fisher's exact test. Two-sided $P$ values were always computed and differences were considered statistically significant at $p$ $\leq 0.05$. All analysis performed using satatistical package for social solution (SPSS) software version 17 .

\section{Results}

The two groups were similar according to clinical characteristics such as age, risk factors, gender, smoking, using alcohol, BMI, previous myocardial infarction, dyslipidemia, Previous cerebrovascular accident, EuroSCORE and history of congestive heart failure (Table 1). There were longer aortic $x$-clamp time (mean \pm SD) and total bypass time (mean \pm $\mathrm{SD})$ in group I versus group II. $(62 \pm 18,62.1 \pm 16.0, p \leq 0.05)$
(Table 2). There were found more LMCAd in group I versus group II (although this difference did not reach statistical significance, $p \geq 0.05$ ), and group I patients were more likely to have had manifestation of coronary artery disease (mean \pm SD) $(3.15 \pm 1.6$ versus $2.8 \pm 1.4, p \leq 0.05)$ and to have significant internal carotid artery velocity more than group I to group II ( $(\mathrm{cm} / \mathrm{sec})$ mean \pm SD) $160 \pm 60$ versus $140 \pm 50, p \leq$ $0.05)$, although this difference was no longer significant when only the rate of previous stroke or TIA was considered and carotid artery occlusion (only ICA velocity) Table 1. Group I also had a trend toward higher prevalence of significant left ventricular dysfunction (LVEF mean \pm SD $45.8 \pm 13.65$ versus $48.9 \pm 11.43 p \leq 0.05)$. Coronary artery lessions have more affected Group I than Group II and number of coronary artery disease have more Group I rather than Group II (Table 3). Coronary angiographic have been evaluating Group $C$ lessions more affected group I than group II ( $p \leq 0.05$ ) (Table 3$)$. There haven't seen mediastinitis in two groups. There have wound infections in 3 patients group I and 2 patients in Group II but there haven't statistical differrentation. These have wounded infection limitation area (3 patients have safen insizyon line, 1 patient sternal mediastinal area and one patient thorax tube line). All of the patients have heeling after 2 weeks postoperative period (Table 2).

Table 2: Operative variables results.

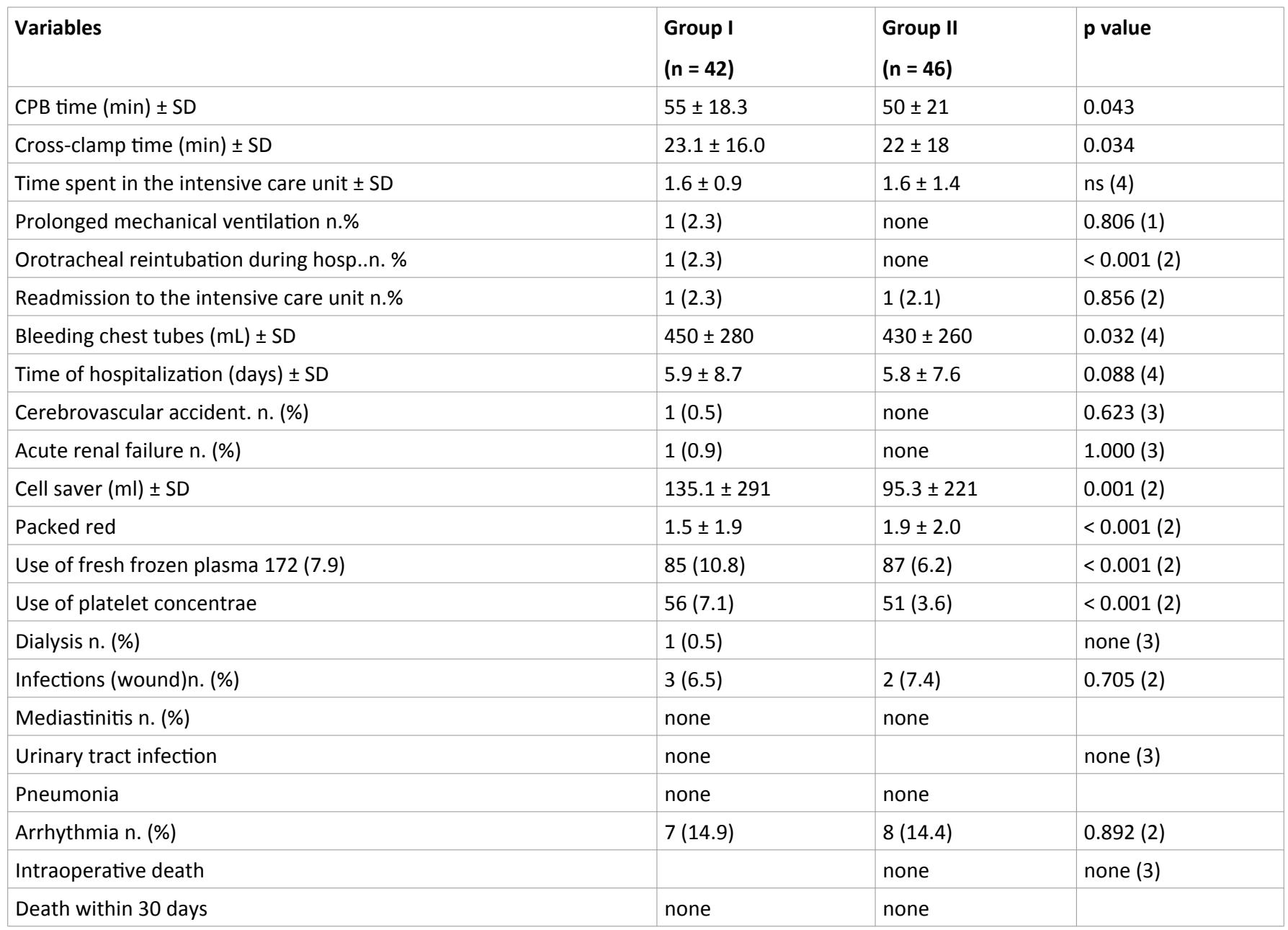

(1) Descriptive level of probability by the Student's t-test; (2) Descriptive level of probability by the chi-square test; (3) Descriptive level of probability by the Fisher's exact test; (4) Descriptive level of probability by the nonparametric Mann-Whitney U-test. 
Citation: Yasa H (2020) Prognosis and Complications of Hyperuricemia Patients Undergoing Isolated Coronary Artery Bypass Surgery. J Cardiothorac Surg Ther 2020:4(1):61-60

Table 3: Preoperative angiographic characteristics.

\begin{tabular}{|c|c|c|c|}
\hline & Group I & Group II & $p$ Value \\
\hline \multicolumn{4}{|c|}{ Coronary lesions. (AHA classification) } \\
\hline Type A & 8 & 12 & NS \\
\hline Type B & 20 & 21 & NS \\
\hline Type C & 14 & 11 & 0.012 \\
\hline Single vessel & 10 & 14 & 0.023 \\
\hline Double vessel & 14 & 16 & NS \\
\hline Triple vessel & 18 & 14 & NS \\
\hline \multicolumn{4}{|c|}{ Left main coronary artery } \\
\hline Disease & 1 & 4 & 0.011 \\
\hline \multicolumn{4}{|l|}{ Ejection fraction } \\
\hline (mean \pm SD) (\%) & $43 \pm 10.4$ & $45 \pm 11.7$ & NS \\
\hline \multicolumn{4}{|c|}{ Significant left ventricular } \\
\hline Dysfunction & 25 & 22 & 0.031 \\
\hline
\end{tabular}

AHA: American Heart Association; NS: Not significant

In relation to medical treatment, hyperurisemia had the highest rates of previous use of medications such as calcium-channel blockers (21.5\% of patients with Group I vs. $17.6 \%$ of Group II patients; $P=0.043$ ), angiotensin-converting enzyme inhibitors (52.6\% of patients with Group I vs. $47.4 \%$ of Group II; $P=0.038$ ) and angiotensin II receptor blockers (20.4\% of patients with Group I vs. $15.0 \%$ of Group II; $P=$ 0.048).

\section{Discussion}

Gout is a painful inflammatory arthritis which can eventually lead to the decrease in quality of life [11]. It is also a risk factor for mortality and cardiovascular morbidity [12]. The prevalence is reported to be increasing in many countries. In the USA the figure reached as much as $3.9 \%$ (8.3 million inhabitants) during 2007-2008 [8]. IGout affects approximately $2 \%$ of men older than 30 -years and $2 \%$ of women older than 50 -years, and is the most common form of inflammatory joint disease in men older than 40-years. Serum uric levels are, on average, 0.5 to $1.0 \mathrm{mg} / \mathrm{dL}$ higher in men than women, making male sex a risk factor for hyperurisemia and gout. Lower serum uric levels in women are associated with the presence of estrogen, which is thought to act as an antihyperuricemic [13].

The enzyme, xanthine oxidase, which is present in significant levels only in the liver and intestine, oxidizes hypoxanthine and xanthine to uric acid in the purine catabolic pathway. Xanthin oxidase inhibitors could be used clinically to block the final step in uric acid synthesis, thereby reducing the production of uric acid.

To our knowledge, this is the first studies in a cohort coronary bypass surgery patients admitted to assess whether high uric acid levels at admission might increase risk for major adverse cardiac events during hospitalization, operation variables, using survival approach to describe and analyze the free survivals between hyperurisemic and normouricemic groups.
One study, in 58 patients undergoing complicated cardiac surgery, found that preoperative uric acid $>6.0 \mathrm{mg} / \mathrm{dL}$ was associated with a nearly 4-fold increased risk of Akut kidney Injury (AKI) and a longer hospital stay than preoperative uric acid $\leq 6.0 \mathrm{mg} / \mathrm{dL}$ [14]. Another study, in 190 patients undergoing cardiovascular surgery, found that, after adjustment for confounders by multivariate logistic regression analysis, serum uric acid concentrations $\geq 7.0 \mathrm{mg} / \mathrm{dL}$ were associated with a 35 -fold higher risk of AKI, and increased hospital stay and duration of mechanical ventilation support, than serum uric acid concentrations $<7.0 \mathrm{mg} / \mathrm{dL}$ [15]. Our previous study in patients undergoing cardiovascular surgery also found that preoperative hyperuricemia was an independent risk factor of postoperative AKI and was related to poor outcomes [16]. Our findings are in agreement with those of the above studies. At the same time intubation time, the proportion of reentubation time, time spent in the intensive care, prolonged mechanical ventilation orotracheal reintubation during hospitalization, readmission to the intensive care unit more Group I than Group II. However, no statistical difference was found between the reasons for the low number of cases.

It is very well known that there are certain risk factors such as genetic susceptibility, hypertension, hyperlipidemia, smoking, and diabetes mellitus, which may predispose an individual to coronary artery disease. Additionally, hyperhomocysteinemia and metabolic syndromes have been identified as other risk factors for the disease as well as predictors of the success of the treatment and survival of the patients [11]. Recently, elevated serum uric acid level is identified as an independent risk factor for cardiovascular diseases [13-19]. Several prospective studies have shown an association between hyperuricemia and cardiovascular diseases and death $[1,11-13,15,16]$. In this study, the number of coronary artery involvement in hyperuricemic patients and Type $\mathrm{C}$ lesion in coronary arteries were statistically significant. Asymptomatic long-term follow-up of hyperuricemia is considered to be a factor in this issue. Interestingly, more prevalence of carotid artery disease was observed. Carotid artery disease was not included in this study because of isolated coronary bypass surgery cases. However, velocity flow rates in the USG of preoperative carotid doppler were higher in group I.

Hyperurisemia patients present an increased risk for postoperative infections due to depreciated host defense mechanisms, such as impaired as wound healing and granulocyte function, decreased cellular immunity, impaired complement function, and reduced immune response, which may be influenced by the gout control [3-6].

Another issue is the investigation of hyperuricemia on postoperative bleeding and on the coagulation cascade. In this study, postoperative bleeding rates, red packed and use of fresh frozen were found to be higher in the hyperuricemic group.

The effects of hyperuricemia on kidney function, hypertension and vascular structure are known. The group was mostly bypassed and had a longer lesion length. These noted effects were effective on postoperative bleeding. However, we believe that this issue needs further investigation. 
Citation: Yasa H (2020) Prognosis and Complications of Hyperuricemia Patients Undergoing Isolated Coronary Artery Bypass Surgery. J Cardiothorac Surg Ther 2020:4(1):61-60

Another issue is the question of postoperative use of acetyl salicylic acid. As is known, the use of anti-aging is essential after coronary bypass surgery. Otherwise Low-dose asetylsalicylic acid can precipitate gout attacks. In this case, it is necessary to use clopidogrel, which is another antiagregan in place of acetylsalicylic acid. However, the correlation between clopidogrel and hyperuricemia is not well researched. In this respect, it is also necessary to decide on the study and the application of an antiagregan protocol in postoperative hyperuricemic patients.

Additionally, Gout is more common after surgery, trauma, and dehydration. Certain medications such as diuretics (commonly known as water pills), which treat high blood pressure, that raise the level of uric acid in the bloodstream are risks for gout. For this reason, the hyperuricemic values of patients receiving surgery should be closely monitored. In this study, blood uric acid levels of all patients after the early period were routinely monitored and, if necessary, patients were directed to rheumatology.

Foods and drinks that contribute most to hyperuricemia and gout include: Red meat, organ meat, seafood, beer, liquor, and sugar-sweetened items, especially with high fructose corn syrup. Hyperuricemic diet has been applied in all of your patients and diets have been adjusted by dieticians. As a result of this study, all cardiovascular and cardiologists should be more sensitive about hyperuricemic diet.

Health problems such as obesity, high blood pressure, high blood sugar, and high cholesterol may also lead to increased uric acid and the development of gout. And because some medications may also increase risk. Other causes of hyperuricemia and gout include kidney disease, exposure to lead, hypothyroidism, severe illness or stress, and extreme physical exertion.

As a result, hyperuricemia seems to be an issue that does not stop at all. Hyperuricemia, which has effects on kidney, liver, coronary vessels, carotid and other peripheral vessels, should be considered a metabolic disease, such as a Diabetes Mellitus. Patients with close follow-up and treatment should be informed about possible effects of hyperuricemia. We believe that there should be an entity that should be emphasized especially in terms of cardiovascular surgery.

\section{Disclosure}

The authors have no conflicts of interest to disclose.

\section{Authors Contribution}

Conception \& design of the study. Yasa H. Acquisition of data: Interpretation and analysis of the data, Writing the draft and paper: Yasa $\mathrm{H}$. Agree with manuscript results and conclusions: H.Yasa, Review and approval of final manuscript: H.Yasa.

\section{References}

1. Leyva F, Anker S, Swan JW, et al. (1997) Serum uric acid as an index of impaired oxidative metabolism in chronic heart failure. Eur Heart J 18: 858-865.

2. Kodama S, Saito K, Yachi Y, et al. (2009) Association between serum uric acid and development of type 2 diabetes. Diabetes Care 32: 1737-1742.

3. Nagahama K, Iseki K, Inoue T, et al. (2004) Hyperuricemia and cardiovascular risk factor clustering in a screened cohort in Okinawa, Japan. Hypertens Res 27: 227-233.

4. Krishnan E, Baker JF, Furst DE, et al. (2006) Gout and the risk of acute myocardial infarction. Arthritis Rheum 54: 2688-2696.

5. Ejaz AA, Beaver TM, Shimada M, et al. (2009) Uric acid: A novel risk factor for acute kidney injury in high-risk cardiac surgery patients? Am J Nephrol 30: 425-429.

6. Lapsia V, Johnson RJ, Dass B, et al. (2012) Elevated uric acid increases the risk for acute kidney injury. Am J Med 125: e9-e17.

7. Joung KW, Jo JY, Kim WJ, et al. (2014) Association of preoperative uric acid and acute kidney injury following cardiovascular surgery. Journal of Cardiothoracic and Vascular Anesthesia 28: 1440-1447.

8. Zhu Y, Pandya BJ, Choi HK (2011) Prevalence of gout and hyperuricemia in the US general population: The National Health and Nutrition Examination Survey 2007-2008. Arthritis Rheum 63: 3136-3141.

9. Culleton BF, Larson MG, Kannel WB, et al. (1999) Serum uric acid and risk for cardiovascular disease and death: The Framingham Heart Study. Ann Intern Med 131: 7-13.

10. Fang J, Alderman MH (2000) Serum uric acid and cardiovascular mortality the NHANES I epidemiologic follow-up study, 19711992. National Health and Nutrition Examination Survey. JAMA 283: 2404-2410.

11. Tavil Y, Sen N, Hizal F, et al. (2008) Relationship between elevated levels of serum uric acid and saphenous vein graft disease. Turk Kardiyol Dern Ars 36: 14-18.

12. Suarna C, Dean RT, May J, et al. (1995) Human atherosclerotic plaque contains both oxidized lipids and relatively large amounts of alpha-tocopherol and ascorbate. Arteriosclerosis, Thrombosis, and Vascular Biology 15: 1616-1624.

13. Alderman MH, Cohen H, Madhavan S, et al. (1999) Serum uric acid and cardiovascular events in successfully treated hypertensive patients. Hypertension 34: 144-150.

14. Bos MJ, Koudstaal PJ, Hofman A, et al. (2006) Uric acid is a risk factor for myocardial infarction and stroke: The Rotterdam. Stroke 37: 1503-1507.

15. Tayar JH, Lopez-Olivo MA, Suarez-Almazor ME (2012) Febuxostat for treating chronic gout. Cochrane Database Syst Rev 11.

16. Rathman W, Funkhouser E, Dyer AR, et al. (1998) Relations of hyperuricemia with the various components of the insulin resistance syndrome in young black and white adults: The CARDIA Study. Ann Epidemiol 8: 250-261.

17. Krishnan E, Kwoh K, Schumacher R, et al. (2007) Hyperuricemia and the incidence of hypertension among men without metabolic syndrome. Hypertension 49: 298-303.

18. Leibovici L, Yehezkelli Y, Porter A, et al. (1996) Influence of diabetes mellitus and glycaemic control on the characteristics and outcome of common infections. Diabet Med 13: 457-463.

19. Wallace KL, Riedel A, Ridge NJ, et al. (2004) Increasing prevalence of gout and hyperuricemia over 10 years among older adults in a managed care population. J Rheumatol 31: 1582-1587. 